\title{
Knee Osteoarthritis Severity Level Classification Using Whole Knee Cartilage Damage Index and ANN
}

\author{
Yaodong Du, Juan Shan, Rania Almajalid \\ Department of Computer Science \\ Seidenberg School of CSIS, Pace University \\ New York, NY, USA \\ yd67578n@pace.edu,jshan@pace.edu, \\ ra56319p@pace.edu
}

\author{
Ming Zhang \\ Division of Rheumatology \\ Tufts Medical Center \\ Boston, MA, USA \\ MZhang@tuftsmedicalcenter.org
}

\begin{abstract}
In this study, we extended our previous work on analyzing the relationship between cartilage thickness and osteoarthritis (OA) severity grade change. Cartilage thickness is measured by the Cartilage Damage Index (CDI) which includes 60 points marked on 3D MRI for each knee joint. In our previous work, we used CDI points on femur and tibia compartments only (36 points) as features and employed machine learning methods to predict the OA severity grade change. In this work, we added the 24 CDI points on patella into the feature space and explored whether CDI points from patella could improve the accuracy, on a larger dataset. Kellgren-Lawrence (KL) grade was used to measure OA severity in this study. Artificial neural network (ANN), which showed good performance in our previous study, was employed as the machine learning method. For KL grade classification, experiment results showed that adding patella points improved the performance remarkably, from AUC 0.822 to AUC 0.903 and the whole knee CDI achieved the best classification performance on the dataset.
\end{abstract}

\section{KEYWORDS}

Knee osteoarthritis, Cartilage Damage Index, Machine learning, KL grade

\section{Introduction}

Symptomatic knee osteoarthritis (OA) affects $10 \%$ of the population over the age of 55 years [1], and is a major cause of work loss [2], early retirement [3] and joint replacement [4]. Knee OA carries heavy socioeconomic cost. In 2004, arthritis was estimated to cost the United States $\$ 336$ billion, or 3\% of the gross domestic product, with $\mathrm{OA}$ as the most common form of arthritis [5-6].

\footnotetext{
Permission to make digital or hard copies of part or all of this work for personal or classroom use is granted without fee provided that copies are not made or distributed for profit or commercial advantage and that copies bear this notice and the full citation on the first page. Copyrights for components of this work owned by others than ACM must be honored. Abstracting with credit is permitted. To copy otherwise, or republish, to post on servers or to redistribute to lists, requires prior specific permission and/or a fee. Request permissions from Permissions@acm.org. CHASE '18, September 26-28, 2018, Washington, DC, USA

(C) 2018 Association for Computing Machinery. ACM ISBN 978-1-4503-59580/18/09...\$15.00 https://doi.org/10.1145/3278576.3278585
}

The pathology of OA disease is still unknown and there are no effective interventions that influence its structural progression. The absence of useful image features to detect OA progression is a major technological obstacle to the development of treatment and prevention of knee OA [7]. The advent of magnetic resonance (MR) imaging (MRI) offered the promise of addressing this critical technology gap by allowing quantification of structural damage in joints. However, it is burdensome to measure the cartilage and gain effective quantitative measurements from MR images. For example, it would cost up to 6 hours to manually segment one $3 \mathrm{D}$ knee MRI for a reader without considering the extensive training cost.

In recent studies, a new sensitive cartilage biomarker - cartilage damage index (CDI), was developed and validated by Zhang [8-9]. The method quantifies the cartilage thickness confined to 60 points on the surface of cartilage, increasing the measurement efficiency and scale responsiveness. The CDI was able to detect up to $14.3 \%$ annual cartilage change compared to traditional methods, which detect $2 \sim 3 \%$ only [10].

This work extended our previous work which explored the CDI points on tibiofemoral compartment [11], by adding CDI points on patella into consideration. We used Kellgren-Lawrence (KL) grade as OA severity measure and trained artificial neural network (ANN) to classify the knee joints into different severity classes according to CDI features.

\section{Materials and Methods}

The MR images used in this study were from the Osteoarthritis Initiative, which is a public database initiated to promote the exploration of OA biomarkers. We obtained a sample of 193 pairs of knee (baseline year MR scans) that have complete clinical data. We chose baseline year Kellgren-Lawrence (KL) grade as the measurement to represent OA severity. We divided the data into $\mathrm{KL} \leq 2$ and $\mathrm{KL}>2$ categories, because 2 is a proper threshold to distinguish OA and non-OA cases using KL grade.

Artificial neural network (ANN) was employed to learn the mapping function between the CDI feature space and OA severity. The structure of ANN was composed of an input layer, an output layer, and a hidden layer. The number of neurons contained in the 
hidden layer was determined as (\# of attributes + \# of classes)/2. Backpropagation algorithm was used to update the weights of neurons.

We applied 10-fold cross-validation in training and testing process. The evaluation metrics we used included precision, recall, Fmeasure, Matthew's correlation coefficient (MCC), and the area under the receiver operating characteristic (ROC) curve (AUC). The formulas of these metrics can be found in [11].

\section{Experiment Results}

We used the CDI values on 60 information locations on both lateral and medial compartment as features to classify KL grades. Among the 60 points, 18 are from femur ( 9 lateral femur and 9 medial femur), 18 are from tibia (9 lateral tibia and 9 medial tibia), and 24 are from patella (12 lateral patella and 12 medial patella). Table 1 recorded the performance of different feature combinations and the ROC curves were plotted in Figure 1.

Table 1: Classification Performance of ANN for KL Grade Using Different CDI Combinations

\begin{tabular}{|l|l|l|l|l|l|}
\hline Compartment & Precision & Recall & F-Measure & MCC & AUC \\
\hline Lateral femur tibia & 0.716 & 0.715 & 0.715 & 0.431 & 0.735 \\
\hline $\begin{array}{l}\text { Lateral femur tibia } \\
\text { patella }\end{array}$ & 0.671 & 0.668 & 0.668 & 0.340 & 0.745 \\
\hline Medial femur tibia & 0.676 & 0.674 & 0.672 & 0.348 & 0.717 \\
\hline $\begin{array}{l}\text { Medial femur tibia } \\
\text { patella }\end{array}$ & 0.658 & 0.658 & 0.658 & 0.316 & 0.721 \\
\hline Tibiofemoral & 0.814 & 0.813 & 0.627 & 0.857 & 0.822 \\
\hline Whole knee & 0.830 & 0.829 & 0.829 & 0.659 & 0.903 \\
\hline
\end{tabular}

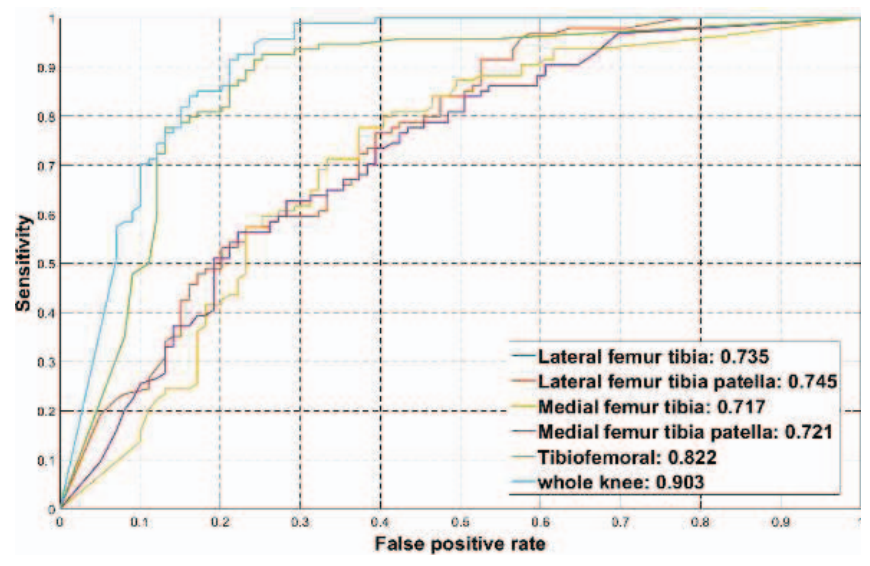

Figure 1: ROC curves on predictions of KL grade by each compartment

As the experiment results showed, adding patella CDI points helped improving the classification performance for both lateral and medial compartments, as well as the whole knee. For lateral compartment, adding patella points improved AUC from 0.735 to 0.745; for medial compartment, adding patella points improved
AUC from 0.717 to 0.721 ; finally, the whole knee (all 60 points) achieved the best classification performance with AUC 0.903, which was improved from AUC 0.822 without adding patella points.

\section{Conclusion}

In this work, we did a pilot study of adding patella CDI points into feature space to classify KL grade for OA diagnosis. This was an extension from our previous work [11] which explored the feature space composed of only femur and tibia CDI points. Different combinations of $60 \mathrm{CDI}$ points on the whole knee (tibia, femur and patella) were used as features and ANN was employed as the classifier. Experiment results showed that the best classification performance $(\mathrm{AUC}=0.903$ ) was achieved using all the $60 \mathrm{CDI}$ points. Particularly, we found that the newly added 24 patella points are helpful in improving performance for KL grade classification. Our future work includes testing the effect of whole knee CDI points to classify other OA severity measures, such as JSN, and running feature selection method to optimize feature space and further improve the classification accuracy.

\section{ACKNOWLEDGMENTS}

This work was supported by NSF SCH-1723420 and NSF SCH1723429.

\section{REFERENCES}

[1] Y. Zhang, J.M. Jordan, "Epidemiology of osteoarthritis," Clin Geriatr Med, vol. 26, 2010, pp. 355-369.

[2] H. Kotlarz, C.L. Gunnarsson, H. Fang, J.A. Rizzo, "Osteoarthritis and absenteeism costs: evidence from US National Survey Data," J Occup Environ Med, vol. 52, 2010, pp. 263-268.

[3] J.P. Raynauld, J. Martel-Pelletier, P. Bias, S. Laufer, B. Haraoui, D. Choquette, A.D. Beaulieu, F. Abram, M. Dorais, E. Vignon, J.P. Pelletier, Canadian Licofelone Study G, "Protective effects of licofelone, a 5-lipoxygenase and cyclo-oxygenase inhibitor, versus naproxen on cartilage loss in knee osteoarthritis: a first multicentre clinical trial using quantitative MRI," Ann Rheum Dis, vol. 68, 2009, pp. 938-947.

[4] L. Murphy, T.A. Schwartz, C.G. Helmick, J.B. Renner, G. Tudor, G. Koch, A. Dragomir, W.D. Kalsbeek, G. Luta, J.M. Jordan, "Lifetime risk of symptomatic knee osteoarthritis," Arthritis Rheum, vol. 59, 2008, pp. 1207-1213.

[5] I.L. Rosemont, United States Bone and Joint Decade. The Burden of Musculoskeletal Diseases in the United States. In Book United States Bone and Joint Decade. The Burden of Musculoskeletal Diseases in the United States (Editor ed.^eds.). City: American Academy of Orthopaedic Surgeons, 2008.

[6] C.R. Chu, A.A. Williams, C.H. Coyle, M.E. Bowers, "Early diagnosis to enable early treatment of pre-osteoarthritis," Arthritis Res Ther, 2012, pp. 14-212.

[7] V.B. Kraus, M. Nevitt, L.J. Sandell, "Summary of the OA biomarkers workshop 2009--biochemical biomarkers: biology, validation, and clinical studies," Osteoarthritis and cartilage, vol. 18, 2010, pp. 742-745.

[8] M. Zhang, J.B. Driban, L.L. Price, D. Harper, G.H. Lo, E. Miller, R.J. Ward, T. McAlindon, "Development of a rapid knee cartilage damage quantification method using magnetic resonance images," BMC Musculoskelet Disord, 2014, pp. $15-264$

[9] M. Zhang, J.B. Driban, L.L. Price, G.H. Lo, E. Miller, T.E. McAlindon, "Development of a Rapid Cartilage Damage Quantification Method for the Lateral Tibiofemoral Compartment Using Magnetic Resonance Images: Data from the Osteoarthritis Initiative," BioMed research international, 2015, pp. 2015-634275.

[10] M. Zhang, L.L. Price, A. Canavatchel, J.B. Driban, P. Yuan, G.H. Lo, T.E. McAlindon, "Cartilage Loss Primarily Occurs in the Most Affected Tibiofemoral Compartment with no Evidence of a Ceiling Effect among Advanced-Stage Disease: A Two-year Longitudinal Study of Data from the Osteoarthritis," Arthritis \& Rheumatololgy, vol. 68, 2016, (suppl 10). 
Knee Osteoarthritis Severity Level Classification Using Whole Knee Cartilage Damage Index and ANN

[11] Y.D. Du, J. Shan, and M. Zhang, "Knee Osteoarthritis Prediction on MR Images Using Cartilage Damage Index and Machine Learning Methods," Proc. IEEE International Conference on Bioinformatics and Biomedicine (BIBM 2017), 2017, pp. 671-677.
CHASE '18, September 26-28, 2018, Washington, DC, USA 